\title{
Theme Issue on Interactive Mobile Information Access
}

\author{
Fabio Crestani \& Mark Dunlop, University of Strathclyde, Scotland \\ Matt Jones \& Steve Jones, University of Waikato, New Zealand \\ Stefano Mizzaro, University of Udine, Italy
}

The volume and forms of information that mobile devices can access, store and disseminate continue to develop at a rapid pace, and many devices provide multiple connectivity mechanisms such as infra-red, short- and wide-area wireless networking and telephony. Yet for most owners of mobile devices today, information access means downloading news snippets, music, ring-tones, weather reports, movie listings and so on.

In this issue of Personal and Ubiquitous Computing we look to more sophisticated notions of mobile information management, considering a dynamic integration of mobile devices into everyday activities and environments, be they related to work or leisure, individual or group oriented, predictable or serendipitous.

We present a selection of papers that examine issues relating to a diversity of user groups and environments, with a central theme of accessing information whilst on the move. In addition to seeking contributions via the Call for Papers, participants in the Mobile and Ubiquitous Information Access Workshop at the Mobile HCI 2004 Conference were invited to make submissions. Sixteen papers were initially submitted by authors from eight countries: UK, Canada, Sweden, Denmark, USA, Germany, Taiwan and Austria. Each paper was considered by at least three members of the review committee, leading to the conditional acceptance of the six papers included in the issue. These papers were then revised by the authors and subjected to further consideration by the editors prior to final revision and acceptance.

The papers reflect the increasing importance of systems that accommodate contextual factors such as location and integrate with external resources such as documents and objects in the physical environment. A range of research approaches is also illustrated, from qualitative ethnographic styles to statistical modelling.

The first two papers investigate the introduction of tailored mobile information systems into existing professional work environments and processes. Esbjörnsson discusses PlaceMemo, a system that helps road inspectors to record defects and locate them again at a later date. These users are highly mobile, travelling more than 200 kilometres each day, with a need to associate reports with geographic locations, to do so quickly, and without diverting attention from the task of driving safely. The paper shows the utility of lightweight interaction to record voice memos that are automatically associated with a GPS location, and the automated replay of memos prior to arrival at a location of interest. The system was generally well received for use in the field, but the paper also highlights the difficulties experienced by users when integrating the captured information with standard recording and reporting processes, and how user needs changed when they were not mobile.

Skov and Høegh describe the design and deployment of MOBILEWARD, a system to support nurses in carrying out their morning rounds in a hospital ward. The system makes use of available contextual information (a nurse's location, patients at a nurse's location and the current time) to dynamically push important information to handheld devices carried by the ward staff. The goal for the system is to overcome the unpredictability of the nurses' information needs, redundant recording of patient-related data, and the potential ambiguity of handwritten notes. Laboratory and field studies produced interesting insights: users were confused by automatic changes to the interface as the system identified changes in context; some users were concerned about validation of information stored in the system because of 
the potential impact of errors; and that along with information the mobile device could carry bacteria between locations.

The next two papers apply mobile technologies to information provision and access in differing leisure contexts: tourist navigation and shopping. Reilly et al.'s Marked-up Maps system illustrates an interesting blend of physical and digital information resources-physical maps are augmented with RFID (Radio Frequency Identification) tags that a handheld device and RFID reader recognise. By holding the device above an area of interest on a map (such as a user's foldable street map, or a wall-affixed subway map) relevant information is retrieved and displayed on the device. A study revealed generally positive user responses, although the nature of the map under consideration was found to impact upon the technique's effectiveness. Concluding that the concept has potential, Reilly et al suggest interesting avenues for future research.

Kurkovsky and Harihar describe a context-aware mobile commerce system call SMMART, which alerts users to products of interests when they visit retail stores. As a mobile recommender system SMMART exploits the user's interest profile, browsing patterns, physical location and retailer's product database to provide relevant, personalised and context dependent notifications of potentially interesting products and offers. As such it takes the recommendation model that is widely adopted by on-line retailers and places it in a physical retail environment where the consumer can see, touch and test the recommended products. Users of the prototype system were very positive about its potential.

Establishing a user's location can help to identify what information the user may need or have and interest in, and the most appropriate manner in which that information can be presented. Smith et al. examine how a user's acoustic environment can be analysed to determine both the social and physical contexts in which they are using a mobile device. They explain how audio samples can be used to build audio profiles of environments (e.g. car, office, supermarket) such that they can be recognised at a later date with a high degree of accuracy even on devices with limited processing capabilities. Information can then be presented to the user via the most appropriate output modality for the context, be it voice, graphic or text.

Finally, Eagle and Pentland present initial results from an ongoing long-term and large-scale study of mobile phone use, in which the devices are 'curious' about a user's behaviour and context. 100 participants were provided with phones that reported use of the phone and onboard applications and also location and proximity information with respect to other users. The paper illustrates how the richness of the captured information can provide insight into how devices are used for information access and other services, the nature of inter-personal relationships, and both individual and group behaviours.

\section{Acknowledgements}

We would like to thank Peter Thomas (Editor-in-Chief) for inviting us to develop this theme issue and the members of the review committee for their work: Akseli Anttila, Russell Beale, Nick Bryan-Kinns, George Buchanan, Keith Cheverst, Andy Cockburn, Oscar De Bruijn, Vincenzo Della Mea, Luca Di Gaspero, Jonathan Foote, Richard Harper, Annika Hinze, Gareth Jones, Mun-Kew Leong, Kent Lyons, Robert Macredie, Natasa Milic-Frayling, Andreas Paepcke, Dynal Patel, Jörg Roth, Ivan Scagnetto, Ed Schofield and Barry Smyth, 